\title{
GESTÃO DE PROJETO COM FOCO EM CONTROLE DE MANUTENÇÃO UTILIZANDO A FERRAMENTA CURVA S
}

DOI 10.37619/issn2447-5378.v7i1.329.20-26

\author{
R.P. Santos ${ }^{1}{ }^{*} ;$ S.C. Nogueira ${ }^{1}$; N.E. Yamada ${ }^{1}$ \\ 1 Faculdade de Tecnologia de São José dos Campos - Professor Jessen Vidal \\ Av. Cesare Mansueto Giulio Lattes, 1350 -Eugênio de Melo, São José dos Campos/SP, \\ CEP.: 12247-014, Brasil. \\ Telefone: (12) 3905-2423 \\ *rafaela.santos28@fatec.sp.gov.br
}

\begin{abstract}
RESUMO: O planejamento da manutenção possui um conjunto de conhecimentos que são de grande importância para o gerenciamento das atividades de manutenção dentro das empresas. Dentre as ferramentas existentes para o planejamento da manutenção industrial, destaca-se a Curva $S$ que se trata de um dispositivo amplamente utilizado pelas empresas, uma vez que permite realizar um acompanhamento da execução da manutenção, segundo o planejamento previamente consolidado. Baseado nas considerações feitas, este trabalho tem como objetivo apresentar a importância do planejamento da manutenção sobre as atividades executadas em uma empresa, onde para tanto foi realizado um estudo de caso junto a empresa REVAP - Refinaria Henrique Lage avaliando como a empresa utiliza a curva S para controlar a execução de manutenção de seus painéis de iluminação e de como o planejamento da manutenção é o centro de uma organização que funciona bem.
\end{abstract}

PALAVRAS-CHAVE: Planejamento; Manutenção; Curva S.

A B S T R A C T: Maintenance planning has a set of knowledge that is of great importance for the management of maintenance activities within companies. Among the existing tools for industrial maintenance planning, the S-Curve stands out, which is a device widely used by companies, since it allows monitoring the execution of maintenance, according to previously consolidated planning. Based on the considerations made, this work aims to present the importance of maintenance planning over the activities performed in a company, where a case study was carried out with the company REVAP - Refinaria Henrique Lage evaluating how the company uses the S curve to control the execution of maintenance of its lighting panels and how the maintenance planning is the center of an organization that works well.

KEYWORDS: Planning; Maintenance; S Curve.

\section{INTRODUÇÃ̃O}

\subsection{Objetivo}

O planejamento de manutenção identifica o quê, o porquê e o como? Estas três indagações permitem que o planejador identifique os problemas e forneça as informações e materiais para evitálos. Um planejamento de manutenção bem-sucedido se define quando se conhece e se garante que as atividades de manutenção sejam gerenciadas e executadas dentro das necessidades dos processos envolvidos na manutenção (COSTA, 2013). 


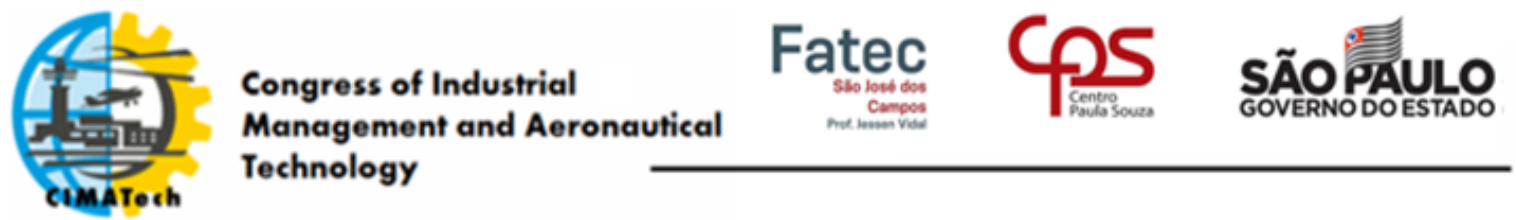

ISSN $2447-5378$

A maioria dos problemas enfrentados pela equipe de planejamento da manutenção está relacionada com a falta de critérios para se definir claramente os materiais específicos para serem usados e aqueles necessários para fazer o trabalho, bem como para se identificar se a tarefa pode ser concluída com o uso de ferramentas manuais básicas ou se necessita do uso de ferramentas especiais.

O planejamento da manutenção fornece o contexto para o indivíduo que está realizando a parte operacional da manutenção, para que ele entenda por que está realizando essa tarefa específica. Tal planejamento não precisa necessariamente ser complexo, porém abrangente e objetivo. (KARDEC; NASCIF, 2009).

Neste sentido, o planejador é capaz de identificar e fornecer desenhos, especificações ou outra documentação que possa ser útil para o ofício.

\subsection{Metodologia}

Dentro do planejamento da manutenção, muitas ferramentas podem ser utilizadas para se avaliar e se tomar decisões dentro das atividades de manutenção de uma empresa. Dentre as ferramentas existentes, destaca-se a Curva $\mathrm{S}$ que se apresenta como uma alternativa muito utilizada e de grande valia para o sucesso da manutenção industrial.

Considerando-se a árdua corrida das empresas pela busca da eficiência e de perfeição, acreditase que quanto mais aperfeiçoados são os processos, maior é a lucratividade da empresa. Partindo deste pressuposto, o objetivo deste trabalho consiste em aplicar a ferramenta Curva S para uma análise das questões relacionadas ao planejamento e ao controle de processos voltados para a manutenção. Para este estudo foram utilizados dados reais para analisar a importância da utilização desta ferramenta de forma visual e eficaz, que tem como finalidade acompanhar o andamento de projetos, auxiliando na tomada de decisões e na visualização de desvios em um determinado processo.

\section{EMBASAMENTO TEÓRICO}

\subsection{Planejamento da Manutenção (PM)}

PM pode ser definido como um processo completo que identifica e soluciona quaisquer problemas possíveis com antecedência. Isto envolve identificar peças e ferramentas necessárias para os trabalhos e garantir que eles estejam disponíveis e dispostos nas áreas apropriadas, pedir que um planejador escreva instruções sobre como concluir um trabalho e até mesmo determinar e reunir as peças e / ou ferramentas necessárias antes que um trabalho seja atribuído (XENOS, 1998).

O PM deve definir "o quê", "por que" e "como". Isso significa especificar o que e com quais materiais, ferramentas e equipamentos precisa ser feito o trabalho; por que uma ação específica foi escolhida (por que uma válvula está sendo substituída em vez de uma sede); e como o trabalho deve ser concluído. Dessa maneira, o PM objetiva-se a determinar os trabalhos de manutenção corretos e prepará-los para o agendamento.

Para fazer isso, um planejador designado desenvolve um plano de trabalho para cada solicitação de trabalho. Esses planos de trabalho detalham tudo o que um técnico deve fazer e usar para realizar a tarefa (VIANA, 2006).

Existe um grande valor no planejamento da carga de trabalho de manutenção em um nível macro em um horizonte de longo prazo, bem como em um nível detalhado em um horizonte curto. Essas duas atividades servem para propósitos significativamente diferentes. Ordens de serviço regulares são criadas automaticamente a partir dos modelos de ordem de serviço na tabela de manutenção. Essas novas ordens de serviço são geradas normalmente dias antes da data de início de 


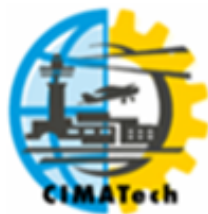

destino especificada no PM. Outras ordens de serviço também são criadas manualmente pelos usuários do sistema (TAVARES, 2000).

\subsection{A Curva S}

A curva $S$ refere-se a um gráfico usado para descrever, visualizar e prever o desempenho de um projeto ou horas extras de atividades. É uma curva logística que plota o progresso de uma variável relacionando-a com outra variável ao longo do tempo. O termo Curva $\mathrm{S}$ foi desenvolvido como resultado da forma que os dados assumem. Projetos na Curva $\mathrm{S}$ geralmente experimentam um crescimento lento no início e no final, ajudando a descrever e prever o desempenho de uma variável ou empresa durante um período de tempo (KARDEC; NASCIF, 2009).

A curva s costuma formar a forma de um "s" porque o crescimento do projeto nos estágios iniciais geralmente é lento, onde a atividade em questão ainda está em seu início de execução, onde os membros da equipe estão pesquisando o setor ou apenas começando a se engajar na primeira fase da execução, que pode levar mais tempo a princípio, antes que eles entendam o problema ou antes que haja problemas para resolver (COSTA, 2013).

Então, à medida que mais progresso é feito, o crescimento acelera rapidamente, criando a inclinação ascendente que forma a parte do meio do "s". Esse ponto de crescimento máximo é chamado de ponto de inflexão. Durante esse período, os membros da equipe do projeto estão trabalhando intensamente no projeto, e muitos dos principais custos do projeto são incorridos. Após o ponto de inflexão, o crescimento começa a se estabilizar, formando a parte superior do "s" conhecido como assíntota superior - e a fase "madura" do projeto. Isso ocorre porque o projeto está concluído principalmente neste ponto e está acabando: Normalmente, apenas tarefas como retoques finais e aprovações finais são deixadas nesse momento (VIANA, 2006).

\section{DESENVOLVIMENTO DA TEMÁTICA}

De acordo com a proposta definida para este estudo, foi realizada uma análise do planejamento de manutenção do sistema de iluminação de uma empresa, onde será avaliada a Curva $\mathrm{S}$ que determina a evolução da manutenção de tal sistema. A empresa escolhida para a realização deste estudo foi a Refinaria Henrique Lage (REVAP) que está localizada na Rodovia Presidente Dutra, em São José dos Campos, no Vale do Paraíba.

O cenário analisado para a realização deste estudo de caso se apoiou nas informações fornecidas pela empresa sobre o planejamento de manutenção desenvolvido pela mesma para a manutenção de seus painéis de iluminação, que se encontram espalhados por toda a planta em suas várias unidades de operação. A distribuição dos painéis dentro da empresa pode ser melhor entendida visualizandose a Tabela 1 .

Tabela 1 - Distribuição dos painéis de iluminação da REVAP.

\begin{tabular}{|c|c|c|c|c|c|c|c|c|c|c|c|c|c|c|c|c|c|c|c|c|c|}
\hline 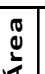 & \multicolumn{3}{|c|}{$\mathrm{DH}$} & \multicolumn{3}{|c|}{ DCCF } & \multicolumn{2}{|c|}{ COQUE } & \multicolumn{8}{|c|}{ HRC } & \multicolumn{5}{|c|}{ ENERGIA } \\
\hline 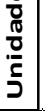 & $\mathrm{U}-210$ & U-272 & U-292 & U-220 & U-274 & U-683 & U-276 & U-591 & U-230 & U-222 & U-311 & U-264 & U-294 & | U-684 & U-262 & U-266 & |U-592 & U-560 & U-515 & U-590 & U-514 \\
\hline 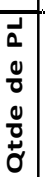 & 15 & 6 & 2 & 10 & 7 & 1 & 11 & 3 & 2 & 3 & 3 & 4 & 2 & 2 & 1 & 2 & 3 & 1 & 1 & 1 & 4 \\
\hline
\end{tabular}

Fonte: REVAP (2020) 


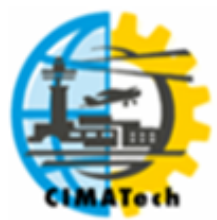

De acordo com as informações prestadas pela REVAP, pode-se visualizar que a empresa possui um total de 84 painéis de iluminação. Para a realização do planejamento de manutenção destes painéis, a empresa adotou como premissas, a estimativa do prazo de manutenção que cada painel levaria em torno de 3 dias para ter sua manutenção efetivamente concluída e a utilização de duas equipes de manutenção para a realização dos serviços.

Com estas premissas em mãos, o PM identificou a necessidade de 252 dias para a realização da manutenção de todos os painéis, onde a utilização de 2 equipes de manutenção diminuiu-se o prazo total dos serviços para uma duração de 126 dias.

Assim, foi elaborado o PM dos painéis da empresa, onde o resultado foi a geração de uma Curva S, ilustrada no Gráfico 1.

Gráfico 1 - Curva S de manutenção dos painéis de iluminação da REVAP

\section{CURVA "S" - ILUMINAÇ̃̃O REVAP Atualizado}

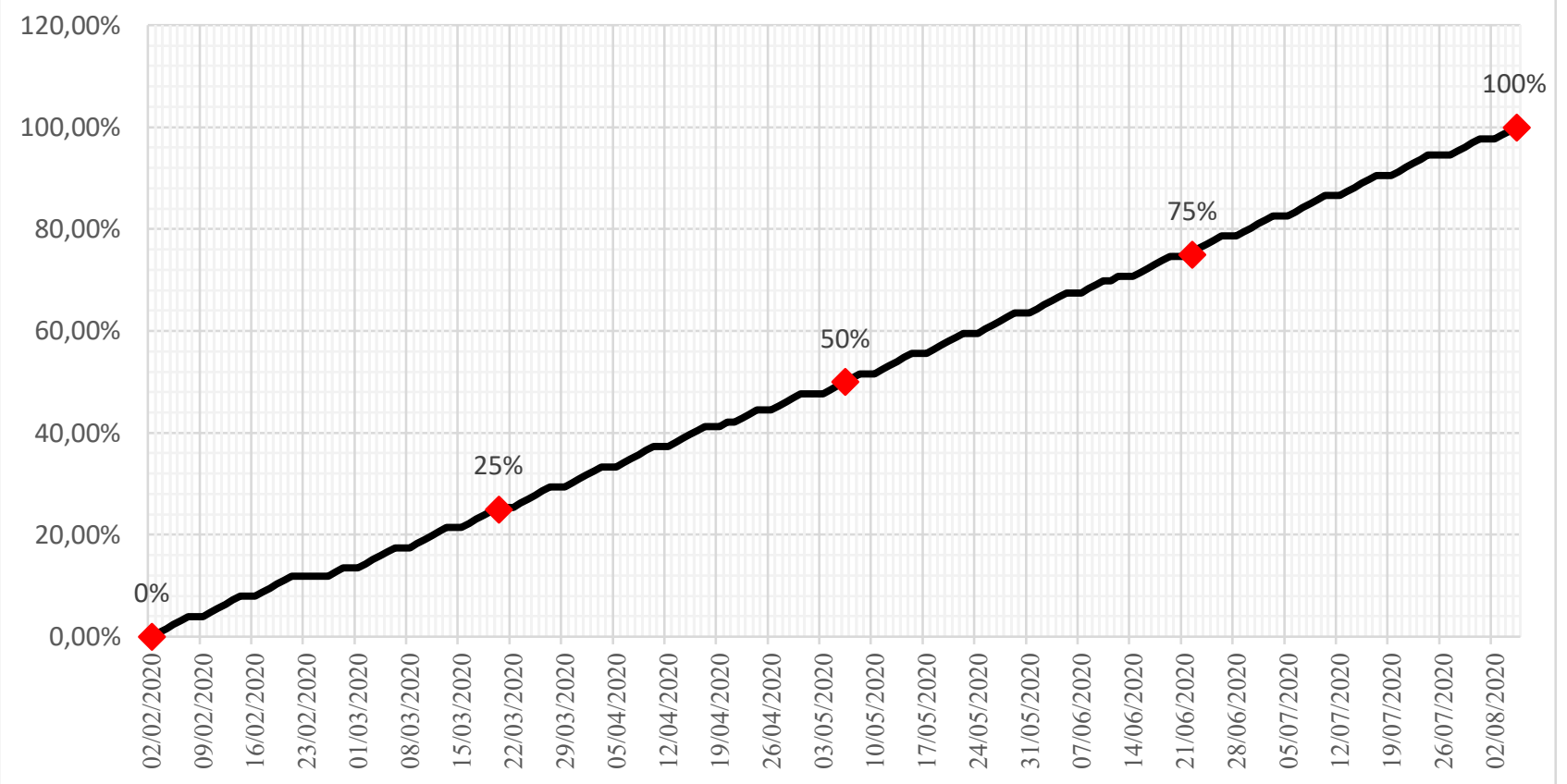

Fonte: REVAP (2020)

Uma vez consolidada a curva de avanço do processo de manutenção dos painéis de iluminação, a empresa colocou em prática o PM após a realização de reuniões com a equipe de manutenção, com o intuito de repassar as metas estabelecidas pelo planejamento e, ao mesmo tempo, de identificar os riscos e oportunidades inerentes à manutenção.

Assim, a empresa reuniu as informações necessárias para balizar as tomadas de decisões durante o processo de manutenção, com o objetivo de garantir que o planejamento formulado seja efetivamente cumprido.

De acordo com as informações disponibilizadas pela REVAP e apresentadas na Tabela 1 acerca do andamento da manutenção realizada nos painéis de iluminação, foram disponibilizados os seguintes resultados de avanço das atividades: 
Tabela 2 - Avanço Físico da manutenção dos painéis de iluminação da REVAP

\begin{tabular}{|c|c|c|c|}
\hline Data & Dia Semana & $\%$ Prevista & $\%$ Realizada \\
\hline $02 / 02 / 2020$ & Dom & $0,00 \%$ & $0,00 \%$ \\
\hline $03 / 02 / 2020$ & Seg & $0,79 \%$ & $1,99 \%$ \\
\hline $04 / 02 / 2020$ & Ter & $1,59 \%$ & $2,38 \%$ \\
\hline $05 / 02 / 2020$ & Qua & $2,38 \%$ & $2,38 \%$ \\
\hline $06 / 02 / 2020$ & Qui & $3,18 \%$ & $2,38 \%$ \\
\hline $07 / 02 / 2020$ & Sex & $3,97 \%$ & $2,38 \%$ \\
\hline $08 / 02 / 2020$ & Sab & $3,97 \%$ & $2,38 \%$ \\
\hline $09 / 02 / 2020$ & Dom & $3,97 \%$ & $2,38 \%$ \\
\hline $10 / 02 / 2020$ & Seg & $4,76 \%$ & $4,76 \%$ \\
\hline $11 / 02 / 2020$ & Ter & $5,56 \%$ & $5,16 \%$ \\
\hline $12 / 02 / 2020$ & Qua & $6,35 \%$ & $5,16 \%$ \\
\hline $13 / 02 / 2020$ & Qui & $7,15 \%$ & $5,16 \%$ \\
\hline $14 / 02 / 2020$ & Sex & $7,94 \%$ & $5,16 \%$ \\
\hline $15 / 02 / 2020$ & Sab & $7,94 \%$ & $5,16 \%$ \\
\hline $16 / 02 / 2020$ & Dom & $7,94 \%$ & $5,16 \%$ \\
\hline $17 / 02 / 2020$ & Seg & $8,73 \%$ & $5,16 \%$ \\
\hline
\end{tabular}

Fonte: REVAP (2020)

Os valores de avanço físico disponibilizados pela REVAP, quando comparados com o planejado na Curva S estabelecida para execução dos serviços, consolida o seguinte cenário executivo mostrado no Gráfico 2.

Gráfico 2 - Curva S de avanço da manutenção dos painéis de iluminação da REVAP

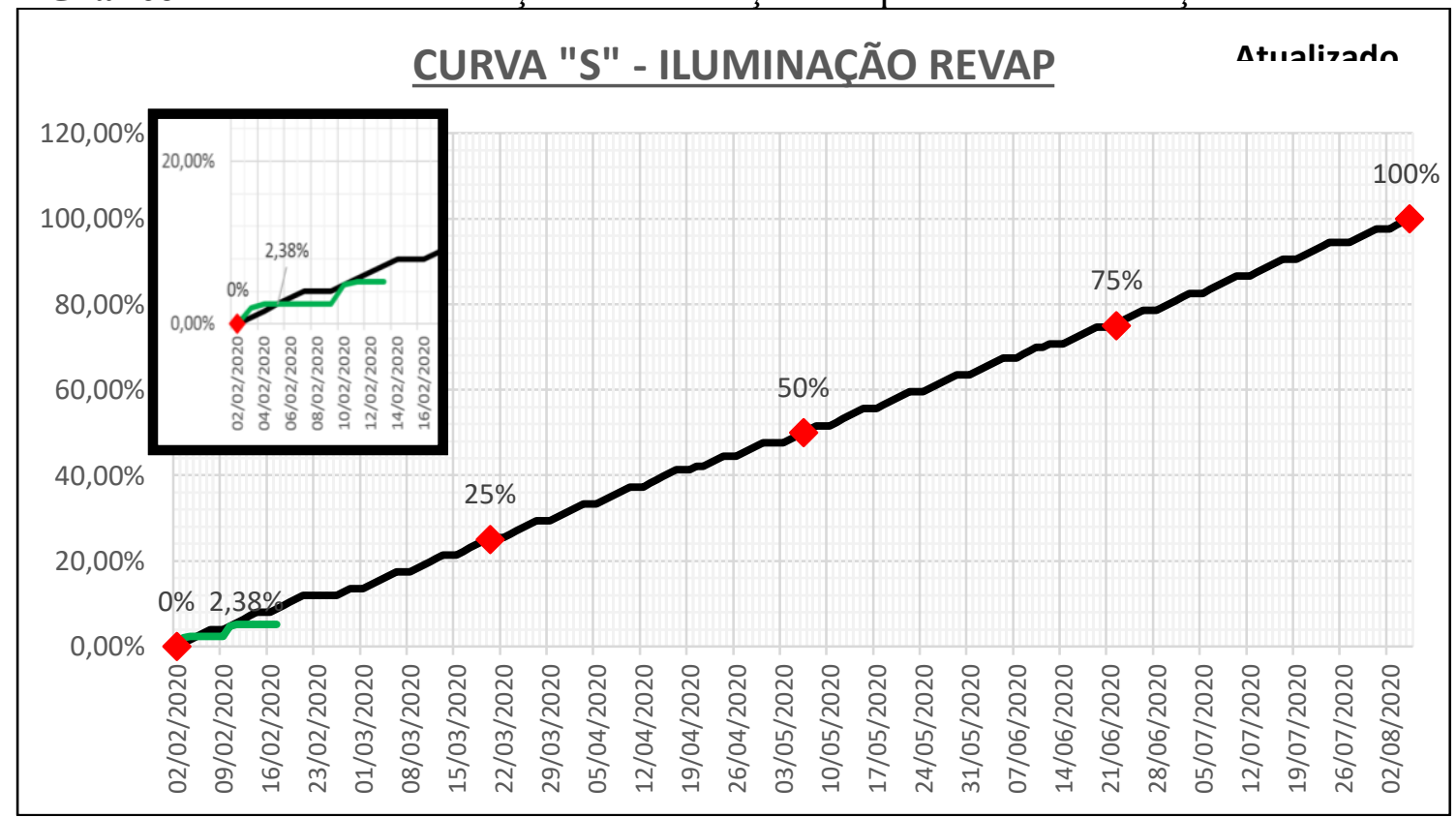

Fonte: REVAP (2020) 


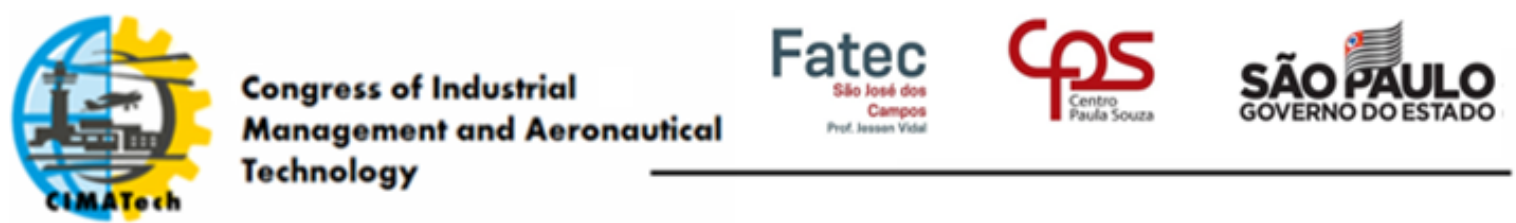

ISSN $2447-5378$

A linha verde representa o avanço da execução, enquanto a linha preta indica o que foi planejado.

\section{RESULTADO E DISCUSSÃO}

De acordo com as informações ilustradas no tópico anterior, pode-se fazer uma análise tanto das premissas de planejamento adotadas para a manutenção dos painéis de iluminação quanto do avanço das atividades dentro da linha de base adotada para os serviços.

Considerando as premissas de planejamento adotadas pela empresa, elas se mostraram como lineares e pouco abrangentes para a organização do planejamento. Isso se deve ao fato de que as informações utilizadas para a elaboração do planejamento da manutenção não contemplam excentricidades dentro do processo de execução, a saber: complexidade dos painéis no tocante ao circuito, mobilização de desmobilização da equipe durante a execução dos serviços, disponibilidade de materiais, dentre outras. A falta destas informações na etapa de planejamento, mesmo que tenham seus pesos contemplados no prazo estimado para cada painel, não permite uma melhor apuração do prazo real que será necessário. Assim, um melhor detalhamento das premissas de planejamento permite para a REVAP um planejamento mais conservador para os painéis.

Como consequência do planejamento realizado pela empresa, tem-se que a Curva S adotada como linha de base dos serviços apresentou um comportamento praticamente linear. Isso porque, ao se estabelecer tempos iguais para os painéis, não torna visível as excentricidades que cada painel pode possuir dentro do processo de manutenção. Assim, a Curva S adotada pela empresa possui um caráter otimista dentro dos resultados previstos para a manutenção.

Já em relação à execução dos serviços de manutenção, pode-se observar que o avanço realizado pela equipe se mostrou abaixo daquele que foi previsto pela linha de base do planejamento. Esse desvio para menos pode ser atribuído tanto pela improdutividade da equipe de manutenção quanto pela linha de base estabelecida, onde a segunda alternativa se mostra com maior impacto. Essa afirmação possui relevância devido ao fato de que, uma vez que as excentricidades dos serviços não foram identificadas, elas provavelmente foram enfrentadas durante a fase de execução, onde o reflexo de tal identificação se apresenta com os resultados consolidados e fornecidos pela empresa.

\section{CONSIDERAÇÕES FINAIS}

De acordo com o estudo realizado neste artigo, pode-se contatar que o planejamento da manutenção é o centro de uma organização de manutenção que funciona bem. Para que o planejamento e a programação da manutenção funcionem, muitos outros sistemas precisam funcionar bem. O mais importante é a inspeção de equipamentos por meio de manutenção preventiva, banco de dados técnicos, como lista de materiais, histórico de ordens de serviço e planos de trabalho padrão. A reposição de peças para a manutenção precisa também funcionar bem.

Pode-se concluir também que as curvas S são ótimas ferramentas gráficas de gerenciamento de projetos para planejar, monitorar, controlar, analisar e prever o status, o progresso e o desempenho do projeto. Elas mostram o progresso do trabalho ao longo do tempo e formam um registro histórico das tendências e variações do projeto. As curvas S podem ser usadas para diferentes propósitos. Alguns dos usos mais comuns das curvas S são para avaliação de progresso e desempenho, conforme o estudo de caso realizado neste artigo.

Diante da apresentação do estudo de caso e dos resultados identificados pelo planejamento da manutenção dos painéis elétricos da empresa REVAP, pode-se constatar que o planejamento dos 
ISSN $2447-5378$

serviços precisa de uma melhoria em sua concepção. Essa melhoria compreende um entendimento mais profundo sobre as excentricidades dos painéis passíveis de manutenção dentro da empresa, onde a particularidade de cada um deve ser levada em conta para que a mesma consiga ter um reflexo direto na Curva S consolidada para os serviços e, consequentemente, delinear um andamento mais realista para os serviços.

Como tomada de decisão a ser tomada pela empresa, mostra-se como ação emergencial um levantamento mais apurado acerca das excentricidades dos painéis que serão submetidos à manutenção, para que sejam conhecidas as particularidades a serem refletidas na Curva S da manutenção. Uma vez levantadas tais excentricidades, deverá ser construída uma nova Curva S para a manutenção, revisando a linha de base inicial para que o cenário de manutenção seja mais realista dentro das necessidades das equipes de manutenção.

Caso as circunstâncias da manutenção não permitam um replanejamento tão apurado como aquele que se mostra necessário, a empresa pode adotar a decisão de aumentar a quantidade de equipes de manutenção para os serviços. Apesar desta decisão garantir uma antecipação dos prazos da linha de base inicialmente consolidada, acarretará o aumento dos custos de manutenção, o que poderia inviabilizar a adoção de tal decisão.

\section{REFERÊNCIAS}

COSTA, M. de A. Gestão estratégica da Manutenção: uma oportunidade para melhorar o resultado operacional. 2013. Trabalho de Conclusão de Curso (Graduação em Engenharia de Produção). Universidade Federal de Juiz de Fora, Juiz de Fora, 2013.

KARDEC, A.; NASCIF, J. Manutenção: função estratégica. Rio de Janeiro: Qualitymark, 2009.

REVAP - REFINARIA HENRIQUE LAGE, 2020.

TAVARES, L. A. Administração Moderna de Manutenção. Rio de Janeiro: Novo Pólo, 2000.

VIANA, H. R. G. Planejamento e Controle da Manutenção. Rio de Janeiro: Qualitymark, 2006.

XENOS, H. G. Gerenciando a Manutenção Produtiva: O Caminho para Eliminar Falhas nos Equipamentos e Aumentar a Produtividade. Rio de Janeiro: EDG, 1998 